\title{
GAINS REALIZED WITH ELECTRONIC OBSERVING AND MEASURING INSTRUMENTS AT THE HALE OBSERVATORIES
}

\author{
Edwin W. Dennison \\ Hale Observatories
}

During recent years it has often been stated that the introduction of automatic electronic equipment can provide two benefits for the astronomical observer. The first is that he can make observations which he could not make with older methods. The second is that he can do the same job more rapidly, more thoroughly, and more efficiently than he had been able to do previously. The first benefit has obviously been realized with many pieces of equipment and I will not concentrate on this point. The second has been a very real one also, and I would like to report on some of the gains which have been made at the Hale Observatories by the introduction of modern electronic equipment. Because of the power which can be brought to bear on astronomical observing problems by the use of digital electronic techniques, almost all of the equipment which I will describe is in one way or another digital in nature.

Digital equipment falls into two broad catagories, the first is the socalled hard-wired digital equipment which consists of digital logic elements which are wired directly to each other and will always perform the same operational task. The relationship between the various elements in a hardwired system may be controlled by means of switches, plug boards, etc., but the variety of operations is always limited by the range of functions which were designed into it originally. The second catagory of digital equipment includes a small computer as its basic controlling element. The computer is coupled with hard-wired peripheral equipment which performs predesigned functions under the control of the computer. The computer itself can be programmed, however, to inter-relate all of the various peripheral devices in an almost infinite number of ways. When adding a new device it is necessary only to build that hardware which relates the new device to the computer, and it is not necessary to redesign or rebuild any of the existing equipment. The computer contains a series of logic elements which can be used to execute various instructions and also contains a memory in which the inter-relation of the instructions are stored. The program which is entered into the memory can be stored on punched paper tape, cards, or magnetic tape and can be changed by the generation of new tapes which are fed into the computer memory and essentially re-configure the entire data or control system.

I would like now to describe three major project areas where these new digital techniques have been used with the observing equipment of the Hale Observatories and indicate the kinds of gains which have been realized by the introduction of these systems. I will also indicate what our general plans are for the future and the gains which we hope to realize in the coming years. 
The first major area of effort was the modernization of the techniques for making direct photometric measurements at the telescope. Initially these measurements were made by means of photomultipliers with DC amplifiers and strip-chart recorders. Because of the problems inherent in DC systems, each measurement ingeneral required at least one minute of telescope observing time and for faint objects this observing time had to be substantially longer in order to compensate for drift in the equipment and the night sky. The time required for the reduction of these strip chart records was frequently a matter of months and an observer occasionally would make a second observing run at the telescope before he had fully reduced the data derived from a previous observing run.

To improve on the photon pulse counting methods which had been introduced by Baum, we started with all solid state digital equipment of the hard-wired type. For each photo-electron which left the photocathode and was multiplied in the dynode structure of the multiplier tube, a single electronic pulse was generated at the anode. This single electron pulse was then amplified and fed to a digital counter and the content of the counter was used as a measure of the total number of photons received during a precisely timed interval. One of the first problems we solved was to make a practical method of digital synchronous detection. With the use of a mechanical chopper which would alternately expose the photomultiplier to sky light and starplus-sky light, it was necessary to generate a count which represented the difference between these two signals. This technique enabled the observers to know immediately the net brightness of the objects which they were measuring and also enabled them to measure extremely faint objects.

Because of the display systems which were used with these datasystems, the observer not only was aware of the rate at which he was accumulating counts in his system, but also was able immediately to check the consistency of several measurements. This could be done easily by estimating the square root of the total number of counts and determining whether the measurements were consistent to within one or two times the RMS standard deviation. By this method the observer not only had an accurate measure of the performance of his equipment, but was also able to detect clouds which were interfering with his observations. The inability to indicate the presence of thin clouds was originally thought to be one of the fundamental objections to pulse counting photometry.

The introduction of the pulse-counting data systems increased the operating effeciency by at least a factor of two. The observers were able to observe twice as many stars per night with this data system, using the same photometers, the same photomultipliers and the same telescopes. Part of this gain came from the fact that for each pulse counting measurement approximately ten seconds of time was required rather than one minute as indicated earlier. The second major gain was that the reduction of the data could be made at a computing center literally in a matter of minutes for each observing run and thus most of the observers who use the computer 
now have their reduced data available to them a day or two after the end of their observing run. Clearly the introduction of these techniques made a very substantial improvement in operating efficiency of the telescopes although they did not provide information which could not have been gathered in other ways.

It should be pointed out that not all observers feel entirely comfortable with these data systems, and as a result, not all observers gain the full advantage. However, all observers one way or another use part of these systems.

The second of our three major areas of effort involves the introduction of automatic controls and data recording equipment for use on the solar magnetograph on the 150 foot solar tower at Mount Wilson. This equipment was designed to scan the image of the sun across the slit of a spectrograph and record the strength of the longitudinal magnetic fields on the surface of the sun. Doppler shift and the intensity of the light was also recorded. Before the use of the digital equipment these measurements were made by means of an analog scanning device. A modulated cathode ray tube was also used at one time to represent the magnetic field strength and polarity. In addition a relatively simple $X-Y$ recorder was used as a means of making magnetogram recordings. The resolution and accuracy of the two $\mathrm{X}-\mathrm{Y}$ display devices was somewhat limited.

With the newer equipment a direct numerical display of the field strength was used for calibration and also for monitoring active areas of the sun. There was provision for recording automatically the output of the data system on magnetic tape. This magnetic tape was then brought to the Computing Center at $\mathrm{Cal}$ Tech and contour maps of the magnetic fields were generated and plotted. Similar contour maps which had been made prior to the introduction of this equipment took from two to four man weeks per magnetogram. The computer, with its plotter, generated the same magnetogram in approximately twenty minutes, thus, once the system was put into operation, the amount of information available about the magnetic field of the sun grew at an enormous rate.

This technique has proven so successful, that we are currently designing and building a complete computer control system which will work on line with the 150 foot tower and will provide for a magnetogram plot immediately after a period of observation, thus reducing the delay between the time of observation and magnetogram plot from several days to twenty minutes or less.

A third area of effort included the construction of automatic data recording systems for the Grant measuring engine, the Sartorius iris photometer and an $\mathrm{X}-\mathrm{Y}$ measuring engine. In all cases encoders were used to translate mechanical position to electrical signals which could be transferred to summary punch cards. In this case the saving of time at the measuring engine was relatively small, but there were gains in recording accuracy and convenience. The greatest single advantage for this kind of instrument 
is that once the data has been prepared for summary punch cards the reduction can be done in minutes at the computing center rather than in weeks by hand computation.

Our future plans include the use of computer data systems directly at the telescopes and for microphotometer and measuring engine applications. In the case of the telescope systems we anticipate gains in better data presentation for the observer who will be able to see his data on a T.V. monitor at the eyepiece location without having to rely on the night assistant. In addition the observer will have at least partial data reduction available to him at the time of observation. In the future we are planning for the time when the observer will have complete data reduction available to him on the day after he completes a night of observations, thus any errors or anomalies in observing can be checked or modified before the next nights observing begins. We also will have automatic dome control, automatic telescope tracking control, thereby minimizing the amount of guiding done by the observer. We are hoping to have automatic telescope positioning by the computer. In this case we envision that the observer or night assistant will enter into the computer the co-ordinates as accurately as he knows them for any particular epoch and then the computer will automatically position the telescope on the object. The computer will make corrections for precession, diffraction, aberration and telescope flexure if necessary. It is our goal to be able to set the telescopes to within ten seconds of arc of any desired object within a time of a few minutes. We have envisioned that savings of ten to twenty percent of observing time by improved telescope setting techniques will provide a very real gain for all telescope operations.

In the case of microphotometer and measuring engines the gains we hope to achieve will be automatic calibration and function transformation of raw data from the machine, immediate data reduction to final desired form, and presumably final recording and plotting of the data in a meaningful format for the observer.

To summarize our experience, it is clear that the introduction of modern digital electronic equipment has not only permitted us to make observations which were previously impossible but also has enabled us to improve subtantially our entire observing process. This latter improvement has come in decreased telescope time for the same observations and because the data is recorded in a format which can be accepted directly by the computing center, the data can now be reduced more thoroughly in a far shorter time.

\section{DISCUSSION}

Vasilevskis: Regarding the iris photometer, what is automated? Only the output or also the centering or is the discrimination automated? Does the observer still have to decide on the opening of the iris? 
Dennison: So far we have automated the output of the iris and the $X, Y$ motion of the carriage. And we are in the process of putting an automatic servo onto the iris, which still has to be built, tested, and finally debugged.

\section{Vasilevskis: And centering of the images?}

Dennison: No, we have no provision at the moment for the automatic centering of the images. So it still requires quite a bit of hand operation.

Strand: With the photometric observations that you mentioned, did you use a solid state detector?

Dennison: No, we used photomultipliers. As yet we've not found a solid state detector with sufficient internal gain to equal a photomultiplier tube. I haven't really heard or seen any in our lab that seems to have sufficient gain. They all seem to have a great deal of noise.

Strand: I would like to make another remark. This is in connection with the operation of our 61-inch astrometric reflector in regard to our parallax program. There we precompute our IBM cards - pardon me for saying that here - our punch cards, send the punch cards to Flagstaff, precessed and corrected for proper motion and so on. And, on the basis of these data on the card, the operator can set the telescope to ten seconds. You don't have the disadvantage of putting a computer in the dome that might create problems of heating.

Dennison: I do not think we have any heating problems the way we are operating our computer, but I realize that this is perfectly possible to do with preprepared sheets. In fact, we are doing that now. But, we should recognize that there are many human problems, many different observers have not always prepared their data when they come to the telescope, this is just an assist to them. 